\title{
Amino acid starvation culture condition sensitizes EGFR-expressing cancer cell lines to gefitinib-mediated cytotoxicity by inducing atypical necroptosis
}

\author{
YU SAITO $^{1}$, SHOTA MORIYA ${ }^{2}$, HIROMI KAZAMA ${ }^{2}$, KAZUHIRO HIRASAWA $^{1}$, KANA MIYAHARA ${ }^{3}$, \\ HIROKO KOKUBA ${ }^{4}$, HIROTSUGU HINO ${ }^{2}$, HIROYUKI KIKUCHI ${ }^{5}$, NAOHARU TAKANO ${ }^{2}$, \\ MASAKI HIRAMOTO ${ }^{2}$, KIYOAKI TSUKAHARA ${ }^{1}$ and KEISUKE MIYAZAWA ${ }^{2}$ \\ Departments of ${ }^{1}$ Otolaryngology (Head and Neck Surgery), ${ }^{2}$ Biochemistry, ${ }^{3}$ Breast Oncology and Surgery, \\ ${ }^{4}$ Joint Research for Basic Medical Science, Institute of Medical Science, and ${ }^{5}$ Preventive Medicine \\ and Public Health, Tokyo Medical University, Tokyo 160-8402, Japan
}

Received November 30, 2017; Accepted December 20, 2017

DOI: 10.3892/ijo.2018.4282

\begin{abstract}
The maintenance of the intracellular level of amino acids is crucial for cellular homeostasis. This is carried out via the regulation of both the influx from the extracellular environment and the recycling of intracellular resources. Since epidermal growth factor receptor (EGFR)-tyrosine kinase inhibitors, including gefitinib (GEF) have been reported to induce the apoptosis of several cancer cell lines, in the present study, we examined whether the cytotoxic effects of GEF are further enhanced under amino acid starvation (AAS) culture conditions. Under AAS culture conditions, the cell killing effect of GEF was synergistically pronounced in the EGFRexpressing cell lines, namely, CAL 27, Detroit 562, A549 and PANC-1 cells compared with those treated with either GEF or AAS alone. The addition of essential amino acids, but not non-essential amino acids to the cell culture medium resulted in the cancellation of this pronounced cytotoxicity. The knockdown of L-type amino acid transporter 1 (LAT-1) by siRNA also enhanced GEF-induced cytotoxicity. Therefore, the shortage of the intracellular amino acid pool appears to determine the sensitivity to GEF. Notably, this enhanced cytotoxicity is not mediated by the induction of apoptosis, but is accompanied by the pronounced induction of autophagy. The presence of necrostatin-1, an inhibitor of receptor-interacting serine/threonine-protein kinase 1 (RIPK-1), but not that of Z-VAD-fmk, attenuated the cytotoxic effects of GEF under AAS culture conditions. Electron microscopy demonstrated
\end{abstract}

Correspondence to: Professor Keisuke Miyazawa, Department of Biochemistry, Tokyo Medical University, 6-1-1 Shinjuku, Shinjuku-ku, Tokyo 160-8402, Japan

E-mail: miyazawa@tokyo-med.ac.jp

Key words: autophagy, necroptosis, apoptosis, gefitinib, epidermal growth factor receptor, cancer, tyrosine kinase inhibitor that the CAL 27 cells treated with GEF under AAS culture conditions exhibited swelling of the cytosol and organelles with an increased number of autophagosomes and autolysosomes, but without chromatin condensation and nuclear fragmentation. Autophagic cell death was excluded as the inhibition of autophagy did not attenuate the cytotoxicity. These results strongly suggest the induction of necroptosis in response to GEF under AAS culture conditions. However, we could not detect any phosphorylation of RIPK-1 and mixed lineage kinase domain like pseudokinase (MLKL), as well as any necrosome formation. Therefore, the enhanced cytotoxic effect of GEF under AAS culture conditions is thought to be mediated by atypical necroptosis.

\section{Introduction}

Amino acids are essential for cellular homeostasis, growth and proliferation via their contribution to a diverse range of cellular processes. Cells use a number of mechanisms to sense and maintain their homeostatic levels. Intracellular levels of amino acids are maintained by the balance between their influx, utilization and recycling (1). The transport of serum amino acids into cells is an active process that is facilitated by plasma membrane-localized amino acid transporters. The members of the L-type amino acid transporter (LAT) family are $\mathrm{Na}^{+}$-independent transporters that deliver neutral amino acids into cells (2). Although the LAT family plays important roles in the development and function of normal tissues, they are frequently increased in cancer cells (3). LAT-1 is most commonly upregulated in multiple types of cancer and has also been used as a biomarker for malignant cancer $(4,5)$. In contrast to the influx of amino acids, the degradation of proteins is also the important intracellular mechanism for releasing free amino acids both under steady-state conditions and during cellular stresses. Macroautophagy (thereafter designated as autophagy) is a self-digestive system conserved in all eukaryotic cells. Cellular proteins and organelles are engulfed into a double-membrane vesicle to form an autophagosome. The cargos are transported to the lysosome and are 
degraded by lysosomal hydrolytic enzymes via membrane fusion between the autophagosome and the lysosome, which is designated as the autolysosome (6). As the recycling of amino acids by this self-digestive mechanism is essential for supplying the intracellular amino acid pool for cellular metabolism, autophagy is markedly upregulated when the cells are exposed to a nutrient-starving condition (7). On the other hand, angiogenesis is indispensable for supporting progressive tumor cell growth. Due to the insufficient vascularization, autophagy is believed to be accelerated as a form of adaptation of tumor cells particularly in the central region of the tumor mass in a hyponutrient and hypoxic microenvironment $(6,8)$.

Autophagy can be potentiated by treatment with chemical inducers or chemotherapeutic agents, as well as in response to amino acid deprivation. We and others have reported that epidermal growth factor receptor (EGFR)-tyrosine kinase inhibitors (TKIs), such as gefitinib (GEF) and erlotinib potently induce autophagy in several cancer cell lines, as well as in murine embryonic fibroblasts (MEFs) (9-13). EGFR is overexpressed or activated in its tyrosine kinase activity by somatic mutation in a broad range of human cancers, including non-small cell lung cancer (NSCLC) $(14,15)$. It has been reported that inactivated endosomal, but not cell surface EGFR interacts with lysosomal-associated transmembrane protein B4 (LAPTM-4B), resulting in a complex formation for recruiting Rubicon from Beclin-1 (Atg6) (16). Since Rubicon is a potent inhibitory protein for autophagy via the molecular interaction with Beclin-1, the dissociation of Rubicon from Beclin-1 results in the initiation of autophagy. Thus, the survival of cancer cells with a higher EGFR expression can be supported by the efficient induction of autophagy under various metabolic stresses (16).

We have also reported that macrolide antibiotics, such as azithromycin (AZM) and clarithromycin (CAM) exert an inhibitory effect on autophagy flux in myeloma and squamous cell carcinoma cell lines $(17,18)$. Of note, AZM and CAM exert cytotoxic effects under amino acid-depleted culture conditions in these cell lines along with the upregulation of the pro-apoptotic transcription factor, $\mathrm{CHOP} / \mathrm{GADD} 153$, although they exhibit no cytotoxicity in complete culture medium (18). Mammalian target of rapamycin (mTOR), is a master regulator that combines amino acid availability to cell growth and autophagy (19-21). Therefore, the shortage of the intracellular amino acid pool appears to determine the sensitivity to various cellular stresses. In this context, it is important to examine whether the effect of GEF is further enhanced when cancer cells are exposed to an amino acid starvation (AAS) culture condition.

Historically, three types of cell death have been identified on the basis of morphological criteria, which include type I (apoptosis), type II (autophagic cell death) and type III (necrosis) (22). Although physiological levels of autophagy are essential for the maintenance of cellular homeostasis during various stress conditions, excessive or uncontrolled levels of autophagy can induce autophagic cell death $(22,23)$. Autophagic cell death was originally defined as a type of cell death accompanied by the large-scale autophagic vacuolization of the cytoplasm and was described during animal development, under tissue homeostasis and in diseased tissues, as well as in cultured cells treated with chemotherapeutic agents or other toxic compounds (22). On the other hand, necrosis has been stereotypically considered as an accidental and passive cell death, as opposed to apoptosis. Necroptosis, in which necrosis is regulated, was originally described in a FADD-deficient variant of human Jurkat $\mathrm{T}$ cells treated with $\mathrm{TNF}-\alpha$, which is characterized as receptor-interacting serine/ threonine-protein kinase (RIPK)1-dependent in association with the morphological features of cell swelling and plasma membrane integrity, but without chromatin condensation and nuclear fragmentation $(24,25)$. Thereafter, non-apoptotic cell death with morphologically necrotic features associated with the cell death inhibition induced by necrostatin 1 (NEC-1), a specific inhibitor of RIPK1, has been 'passively' described as necroptosis.

In this study, we found that the cell killing effect of GEF was apparently pronounced along with the upregulation of autophagy when the cells were cultured under AAS culture conditions plus GEF. Of note, this pronounced effect was not mediated either by the induction of apoptosis or autophagic cell death, but apparently by an atypical type of cell death. The molecular mechanisms and phenotype regarding this atypical cell death are precisely discussed.

\section{Materials and methods}

Reagents. GEF, which was purchased from Cayman Chemical Co. (Ann Arbor, MI, USA), was dissolved in dimethyl sulfoxide (DMSO) to yield $10 \mathrm{mM}$ stock solutions. Z-VAD-fmk, which is a pan-caspase inhibitor, was purchased from Peptide Institute (Osaka, Japan) and 3-methyladenine (3-MA), SP600125, doxycycline hyclate (DOX) and puromycin dihydrochloride were obtained from Sigma-Aldrich (St. Louis, MO, USA). NEC-1, a specific inhibitor of RIPK1, was purchased from Enzo Life Sciences (Farmingdale, NY, USA). Cycloheximide was obtained from Calbiochem (La Jolla, CA, USA). Recombinant human TNF- $\alpha$, staurosporine, amino acid-free Dulbecco's modified Eagle's medium (DMEM) (048-33575), MEM essential amino acids solution (X100; 132-15641) and MEM non-essential amino acids solution (X50; 139-15651) were obtained from Wako Pure Chemical Industries (Osaka, Japan).

Cell lines and culture conditions. The human oral squamous cell carcinoma cell line, CAL 27, the human pharyngeal carcinoma cell line, Detroit 562, the human NSCLC cell line, A549, the human pancreatic cancer cell line, PANC-1, the human colorectal adenocarcinoma cell line, HT-29 and the breast cancer cell line, MDA-MB-231 were obtained from the American Type Culture Collection (ATCC, Manassas, VA, USA). The human NSCLC cell line, PC-9, which carries delE746-A750 in exon 19 of the EGFR gene and exhibits exquisite sensitivity to EGFR TKIs (26), was obtained from the RIKEN BioResource Center (Tsukuba, Japan). The murine embryonic fibroblast (MEF) cell line (DR-wild-type) established by SV-40 immortalization was also obtained from the ATCC. All cell lines were cultured in DMEM plus $10 \%$ fetal bovine serum (FBS; Biosera, Ringmer, UK) and 1\% penicillin/ streptomycin solution (Wako Pure Chemical Industries). The 'AAS' culture conditions described in this study indicate amino acid-free DMEM (048-33575; Wako Pure Chemical Industries) plus $10 \% \mathrm{FBS}$ and $1 \%$ penicillin/streptomycin. 
The m5-7 cell line, an Atg5 tet-off MEF system, was a kind gift from Professor Noboru Mizushima (University of Tokyo, Tokyo, Japan). The m5-7 cells were maintained in DMEM containing $10 \%$ FBS. For the knockdown of the Atg5 gene for the full inhibition of autophagy, the cells were further cultured in the presence of $10 \mathrm{ng} / \mathrm{ml}$ DOX for 4 days (27). All cell lines were cultured in a humidified incubator containing $5 \% \mathrm{CO}_{2}$ and $95 \%$ air at $37^{\circ} \mathrm{C}$. All cell lines were used for the experiments within 10 passages after thawing.

For the typical induction of necroptosis, the HT-29 cells were pre-treated with Z-VAD-fmk $(20 \mu \mathrm{M})$ for $30 \mathrm{~min}$ followed by an additional treatment with cycloheximide $(\mathrm{CHX}$, $10 \mathrm{mg} / \mathrm{ml})$ and human TNF- $\alpha(20 \mathrm{ng} / \mathrm{ml})$ for $8 \mathrm{~h}$ as previously described in the literature (28).

Assessment of cell growth inhibition. Cell growth inhibition was measured by the CellTiter-Blue cell viability assay (Promega, Madison, WI, USA). The cells were treated with or without GEF (5 to $50 \mu \mathrm{M}$ ) for 24 and $48 \mathrm{~h}$ in 96 -well plates (Thermo Fisher Scientific, San Jose, CA, USA) in pentaplicate. During the last $4 \mathrm{~h}$, CellTiter-Blue reagent was added to each well, and fluorescence was measured at $560 \mathrm{~nm}$ excitation and $590 \mathrm{~nm}$ emission. The percentage of the mean fluorescence measured to that in untreated cells and was expressed as \% cell growth inhibition.

Morphological assessment. The cells were spread on glass slides using a Cytospin 4 Centrifuge (Thermo Fisher Scientific) to make glass slide preparations, then stained with May-Grünwald-Giemsa, and examined under a digital microscope (BZ-8000; Keyence Co., Osaka, Japan).

Flow cytometry. For the assessment of apoptosis, the cells were stained with Annexin V and propidium iodide (PI) using the Annexin V-FITC Apoptosis Detection kit (Nacalai Tesque, Kyoto, Japan) according to the manufacturer's instructions and subjected to flow cytometry using the Attune ${ }^{\circledR}$ Acoustic Focusing Cytometer (Life Technologies, Carlsbad, CA, USA).

Immunoblotting. Immunoblotting was performed as previously described (29). Briefly, the cells were lysed with RIPA lysis buffer (Nacalai Tesque) supplemented with a protease and phosphatase inhibitor cocktail (Nacalai Tesque). Cellular proteins were quantified by Bradford assay (Thermo Fisher Scientific). Equal amounts of proteins were loaded onto the gels, separated by sodium dodecyl sulfate-polyacrylamide gel electrophoresis (SDS-PAGE) and transferred onto an Immobilon-P membrane (Millipore, Bedford, MA, USA). The membranes were probed with primary antibodies (Abs), such as anti-microtubule-associated protein 1 light chain 3 (LC3) B antibody (Ab) (NB600-1384; Novus Biologicals, Inc., Littleton, CO, USA, at 1/4,000 dilution), anti-ATG5 Ab (\#12994S, at 1/1,000 dilution), anti-caspase-3 Ab, (\#9662S, 1/1,000), anti-poly(ADP-ribose) polymerase (PARP) Ab (\#9542S, 1/1,000), anti-LAT1 (\#9166S, 1/1,000), anti-RIPK1 (\#4926S, 1/1,000), anti-phospho-RIPK1 (Ser166) (\#65746S, 1/1,000), anti-RIPK3 Abs (\#13526S, 1/1,000) (Cell Signaling Technology, Danvers, MA, USA), anti-mixed lineage kinase domain like pseudokinase (MLKL), anti-phospho-MLKL (Ser358) (ab184718, 1/1,000) Abs (Abcam, Cambridge, MA,
USA) and anti-EGFR Ab (sc-03, 1/1,000), anti-phospho-EGFR (Tyr1173) Ab (sc-101668, 1/1,000), anti-p62 (sequestosome-1) mAb (sc-28359, 1/1,000), anti-GAPDH mAb (sc-32233, $1 / 1,000)$ and anti- $\beta$-actin mAb (sc-47778, 1/1,000) (Santa Cruz Biotechnology, Santa Cruz, CA, USA). Immunoreactive proteins were detected with horseradish peroxidase-conjugated secondary Abs (anti mouse Ab: 115-035-003, at 1/5,000 dilution, anti rabbit Ab: 711-035-152, at 1:5,000 dilution; Jackson ImmunoResearch, West Grove, PA, USA) and an enhanced chemiluminescence reagent (ECL) (Millipore). Densitometry was performed using a Molecular Imager, ChemiDoc XRS system (Bio-Rad Laboratories, Richmond, CA, USA).

Immunoprecipitation. The harvested cells were washed with physiological-buffered saline (PBS) and lysed with lysis buffer (10 mM Tris-HCl, $150 \mathrm{mM} \mathrm{NaCl,} \mathrm{1 \%} \mathrm{NP-40,} \mathrm{pH} 8.0$ ) supplemented with a protease and phosphatase inhibitor cocktail (Nacalai Tesque). The cell lysates were spun down at $16,000 \mathrm{x} \mathrm{g}$ for $15 \mathrm{~min}$. The soluble fraction was collected, and the protein concentration was determined by Bradford assay. Subsequently, $800 \mu \mathrm{g}$ of the extracted protein solution in lysis buffer were immunoprecipitated overnight with either anti-RIPK $1 \mathrm{Ab}$ or anti-RIPK3 $\mathrm{Ab}$ at $4^{\circ} \mathrm{C}$. Antibodies were collected with Protein A SureBeads (Bio-Rad Laboratories) for 1 -h rotation at $4^{\circ} \mathrm{C}$, then washed 3 times with the lysis buffer. The binding proteins on the beads were eluted with $2 \mathrm{X}$ SDS loading buffer by 5-min boiling in a heat block.

Electron microscopy. Following treatment with/without GEF $(25 \mu \mathrm{M})$ under either complete culture condition or AAS culture condition for $24 \mathrm{~h}$, CAL 27 cells were fixed with $2.5 \%$ glutaraldehyde in $0.1 \mathrm{M}$ phosphate buffer $(\mathrm{pH} 7.3)$ for $1 \mathrm{~h}$. The samples were further fixed in $1 \%$ osmium tetroxide for $1 \mathrm{~h}$, dehydrated in graded ethanol (30-100\%), and embedded in Quetol 812 Epoxy Resin (Nisshin EM Co., Ltd., Tokyo, Japan). Ultrathin sections were cut using an Ultracut $\mathbf{J}$ microtome (Reichert Jung, Vienna, Austria). These sections were stained with lead nitrate and uranium acetate (Merck, Darmstadt, FRG) and subjected to electron microscopic analysis using a scanning electron microscope (JEM-1200EX II; JEOL Ltd., Tokyo, Japan).

RNA interference. For the gene silencing of LAT1 and RIPK1 in the CAL 27 cells, the LAT1 siRNA and control siRNA sequences are described as follows: LAT1 sense, GGAAC AUUGUGCUGGCAUUdTdT and antisense, AAUGCCAGCA CAAUGUUCCdTdT; control sense, GUUAAAGGUUUGAC UCGCGdTdT and antisense, CGCGAGUCAAACCUUUAA CdTdT. RIPK1 siRNA (HSS112847) and control siRNA (12935-300) were purchased from Life Technologies (Grand Island, NY, USA). siRNAs were diluted to a final concentration of $10 \mathrm{nM}$ in Opti-MEM I (Life Technologies). Transfection was performed with the cells at $40 \%$ confluency using Lipofectamine RNAiMAX transfection reagent (Life Technologies) according to the manufacturer's instructions. The knockdown efficiency was assessed by immunoblotting.

Stable transfection of $m$ Cherry-EGFP-LC $3 B$ plasmid into A549 and CAL 27 cells. In the present study, a pBABEpuro-mCherry-EGFP-LC3B plasmid vector (no. 22418) was 
purchased from Addgene (Cambridge, MA, USA). The A549 and CAL 27 cells were transfected with this plasmid DNA using Lipofectamine 2000 (Life Technologies) according to the manufacturer's instructions and as previously described (30). Briefly, $4 \mu \mathrm{g}$ of pBABE-puro-mCherry-EGFP-LC3B plasmid solution and $10 \mu 1$ of Lipofectamine 2000 were incubated in $500 \mu$ l of serum-free Opti-MEM (Life Technologies) for $20 \mathrm{~min}$ and mixed into $2 \times 10^{6}$ cells cultured in $1.5 \mathrm{ml}$ of antibiotic-free DMEM with $10 \%$ FBS in a $60-\mathrm{mm}$ dish. At $48 \mathrm{~h}$ after transfection, the cells were seeded into a $150-\mathrm{mm}$ dish with puromycin dihydrochloride (at $2 \mu \mathrm{g} / \mathrm{ml}$ for A549 cells and at $0.5 \mu \mathrm{g} / \mathrm{ml}$ for CAL 27 cells). The individual puromycin-resistant clones were isolated using a cloning ring. After cloning, A549/pBABE-puro-mCherry-EGFP-LC3B and CAL27/pBABE-puro-mCherry-EGFP-LC3B were used for the following experiments.

Time-lapse imaging. On the first day, the A549/pBABEpuro-mCherry-EGFP-LC3B cells (clone \#3) and CAL 27/ pBABE-puro-mCherry-EGFP-LC3B cells (clone \#7) were seeded into the CELLview ${ }^{\mathrm{TM}}$ Cell Culture dish, $35 \mathrm{~mm}$ glass bottom (627860; Greiner Bio-One, Frickenhausen, Germany), and cultured for $24 \mathrm{~h}$ to make them adhere to the glass bottom. On the second day, the cells were washed twice with PBS and incubated in either the AAS culture medium containing $10 \%$ FBS or the complete culture medium containing 10\% FBS with/without GEF $(25 \mu \mathrm{M})$. Thereafter, observation began at 5 -min intervals using a confocal laser scanning microscope (LSM 700; Carl Zeiss, Oberkochen, Germany) for the detection of EGFP at $488 \mathrm{~nm}$ laser wavelength and mCherry at $555 \mathrm{~nm}$ laser wavelength. In this time-series scanning, a 63X oil immersion lens was used, and bright field images were obtained simultaneously. ZEN 2012 Black Edition software (Carl Zeiss) was used for the analysis.

Statistical analysis. All quantitative data are expressed as the means \pm standard deviation (SD). Statistical analysis was performed using a two-tailed non-paired Student's t-test. To assess the synergism of the combined GEF and AAS treatment, multivariate linear regression analysis using both GEF and AAS as independent variables was first performed to determine whether an additive effect was observed between GEF and AAS. Subsequently, interaction terms were added in the model to clarify the existence of a synergistic effect, as previously described (31). The criterion for statistical significance was taken as $\mathrm{P}<0.05$.

\section{Results}

Amino acid starvation enhances GEF-induced cytotoxicity in EGFR-expressing cell lines. It is now well known that the depletion of amino acids in the cell culture medium promotes the induction of autophagy for the adaptation to the shortage of the intracellular amino acid pool (18-21). It has also been reported that EGFR-TKIs, such as GEF induce cytoprotective autophagy in various EGFR-expressing cell lines (9-13). Therefore, in this study, we first examined whether the cytotoxicity of GEF is pronounced under AAS culture conditions using the head and neck cancer cell lines, CAL 27 and Detroit 562, the NSCLC cell lines, A549 and PC-9, and the pancreatic cancer cell line,PANC-1, all of which express EGFR at a higher level, as well as immortalized MEFs (Fig. 1A). All the cell lines were cultured in the presence of GEF at various concentrations in either amino acid-depleted DMEM containing 10\% FBS or complete culture medium for up to $48 \mathrm{~h}$ (Fig. 1B). Normal DMEM contains a sufficient amount of 20 amino acids including $75 \mathrm{mg} / \mathrm{l} \mathrm{L}$-glutamic acid and $30 \mathrm{mg} / \mathrm{l} \mathrm{L}$-methionine, whereas AAS (amino acid-free DMEM plus 10\% FBS) only contains amino acids less than 1/10 of the normal DMEM with 10\% FBS. Although AAS itself exerted some cell growth inhibitory effects, the apparent enhanced cytotoxic effect of GEF was observed even after $24 \mathrm{~h}$ of exposure in the CAL 27, A549 and PANC-1 cells compared to treatment with either AAS or GEF alone. After $48 \mathrm{~h}$ of exposure to GEF under AAS culture conditions, the effect became more apparent in all cell lines except for the PC-9 cells. As previously reported, the PC-9 cells have EGFR mutation with a higher sensitivity to GEF, resulting in a sufficiently strong cell killing effect by GEF alone, which may produce no further enhancement even by combining GEF and AAS $(26,32)$. The PANC-1 cells were resistant to GEF, but exhibited a significant cytotoxicity under AAS culture conditions. After $48 \mathrm{~h}$ of treatment, multivariate linear regression analysis revealed that these pronounced effects were synergistic in the CAL 27, Detroit 562, A549 and PANC-1 cells.

The supplementation of essential amino acids, but not non-essential amino acids, into the AAS culture medium almost cancelled the pronounced cytotoxicity in CAL 27 cells (Fig. 2A). This strongly suggests that the enhanced cytotoxicity by the combined treatment with GEF plus AAS is due to the shortage of the intracellular amino acid pool. To confirm this hypothesis, we subsequently knock down LAT-1 in the CAL 27 cells. It has been reported that among the amino acid transporters, LAT-1 is specifically expressed at a higher level in cancer cells, by which the essential amino acids are imported into cancer cells for supporting cellular metabolism $(3,33)$. As shown in Fig. 2B, the high LAT-1 expression was considerably suppressed by transfection with LAT-1 siRNA in the CAL 27 cells. LAT-1 knockdown resulted in a significant decrease in the viable cell number in the presence of GEF compared with the cells transfected with control siRNA and treated with GEF. Notably, LAT-1 knockdown itself exerted no effect in the absence of GEF, suggesting some metabolic compensation or adaptation for cell survival when the cells were cultured in the complete culture medium.

Sensitization of GEF under AAS conditions is not mediated by the induction of apoptosis. We subsequently examined whether apoptosis is involved in the enhanced GEF-induced cell death under AAS conditions. First, immunoblotting was performed to confirm the cleavage of caspase-3 and PARP as indicators of apoptosis. As shown in Fig. 3A, GEF treatment under complete culture conditions led to some cleavage of caspase-3 and PARP. However, GEF treatment under AAS culture conditions did not lead to any apparent increase in the cleavage of caspase-3 as compared with GEF treatment in complete culture medium, although the cytotoxic effect was pronounced. As shown by flow cytometry, GEF treatment under AAS culture conditions increased the number of Annexin $\mathrm{V}^{+} / \mathrm{PI}^{+}$doublestained cells compared with either GEF treatment under 


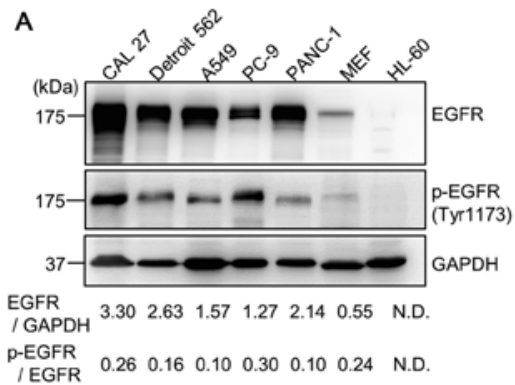

B
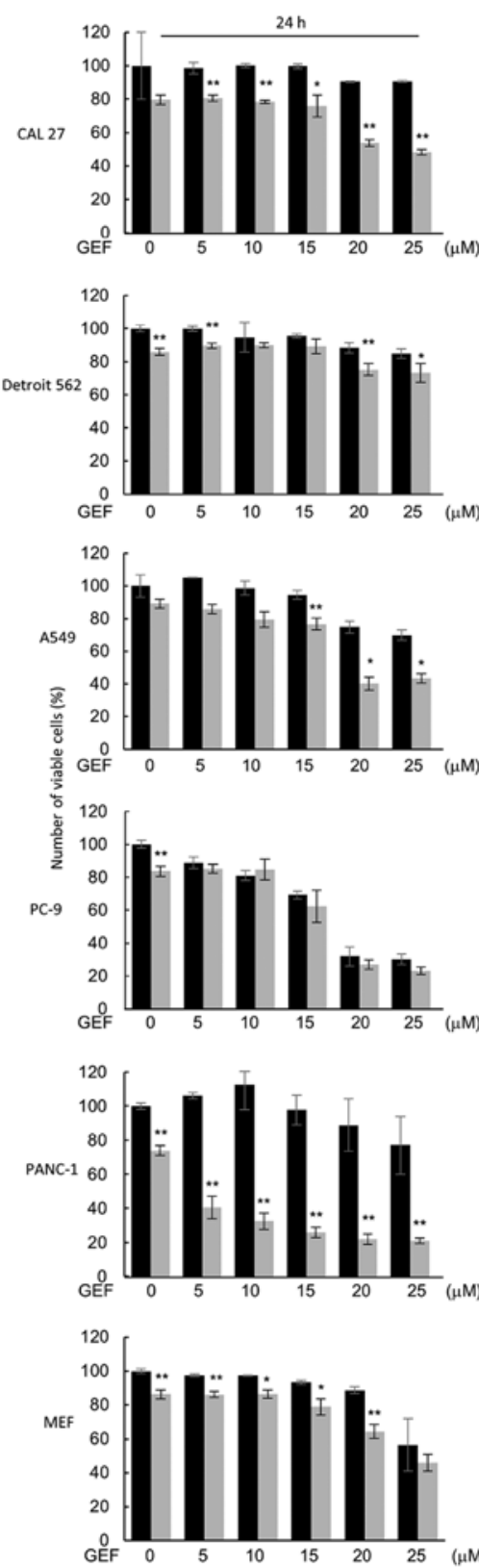
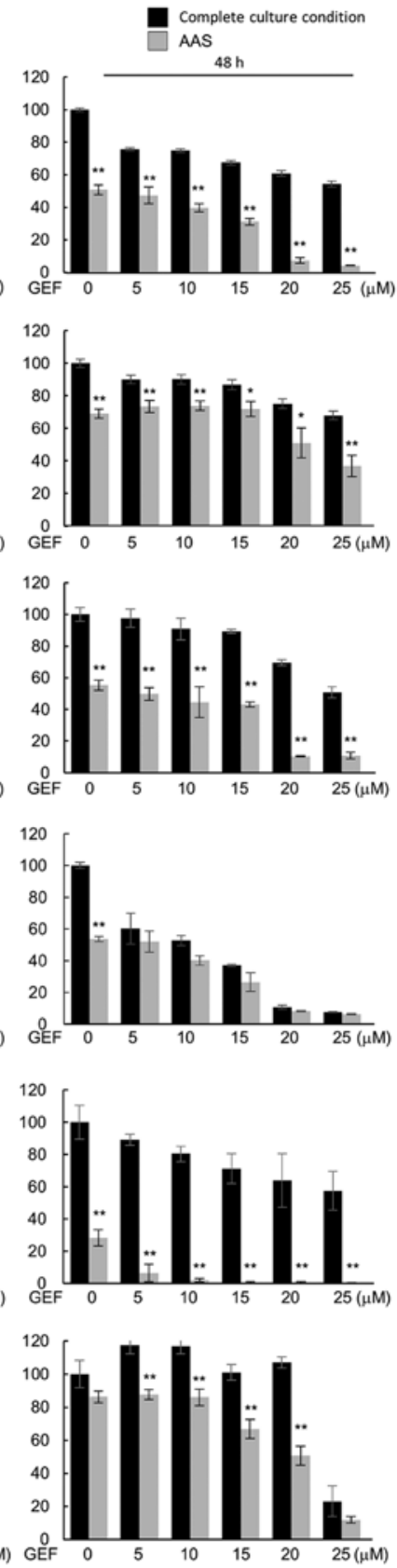

Figure 1. Cell growth inhibition following treatment with gefitinib (GEF) under amino acid starvation (AAS) culture conditions in EGFR-expressing cancer cell lines. (A) Cellular proteins in the CAL 27, Detroit 562, A549, PC-9, PANC-1 and MEF cells in the exponential growth phase in the complete culture condition containing $10 \%$ FBS were lysed as described in Materials and methods. The cellular proteins extracted from the $1 \times 10^{5}$ cells were loaded on each lane and separated by $11.25 \%$ SDS-PAGE and immunoblotted with anti-EGFR antibody (Ab) and anti-phospho-EGFR (Tyr1173) Ab. Immunoblotting with anti-GAPHD mAb was performed as an internal control. The cell lysate of HL-60 leukemia cells was used as a negative control, as previously described (11). Numbers indicate the ratios of EGFR to GAPDH and the ratios of the phospho-EGFR to EGFR in each lane. N.D., not detectable. (B) Cells were cultured under the complete culture condition and AAS culture condition in the presence of GEF $(0-25 \mu \mathrm{M})$ for 24 and $48 \mathrm{~h}$. Viable cell number was assessed by CellTiter-Blue cell viability assay as described in Materials and methods ( $\mathrm{P}<0.05$ and ${ }^{* *} \mathrm{P}<0.01$, complete culture condition vs. AAS by a two-tailed non-paired Student's t-test). By multivariate linear regression analysis using both GEF and AAS as independent variables, the synergistic effect was observed in the $24-\mathrm{h}$ treatment of $\mathrm{CAL}-27$ cells $(\mathrm{P}<0.001)$ and PANC-1 cells $(\mathrm{P}=0.008)$, as well as in the 48-h treatment of CAL-27 cells $(\mathrm{P}<0.001)$, Detroit 562 cells $(\mathrm{P}=0.023)$, A549 cells $(\mathrm{P}<0.001)$ and $\mathrm{PANC}-1$ cells $(\mathrm{P}<0.001)$. In the $\mathrm{PC}-9$ cells, the additive cytotoxic effect was observed in the 48 -h exposure $(\mathrm{P}<0.001)$, but not in the $24-\mathrm{h}$ exposure $(\mathrm{P}=0.335)$. 

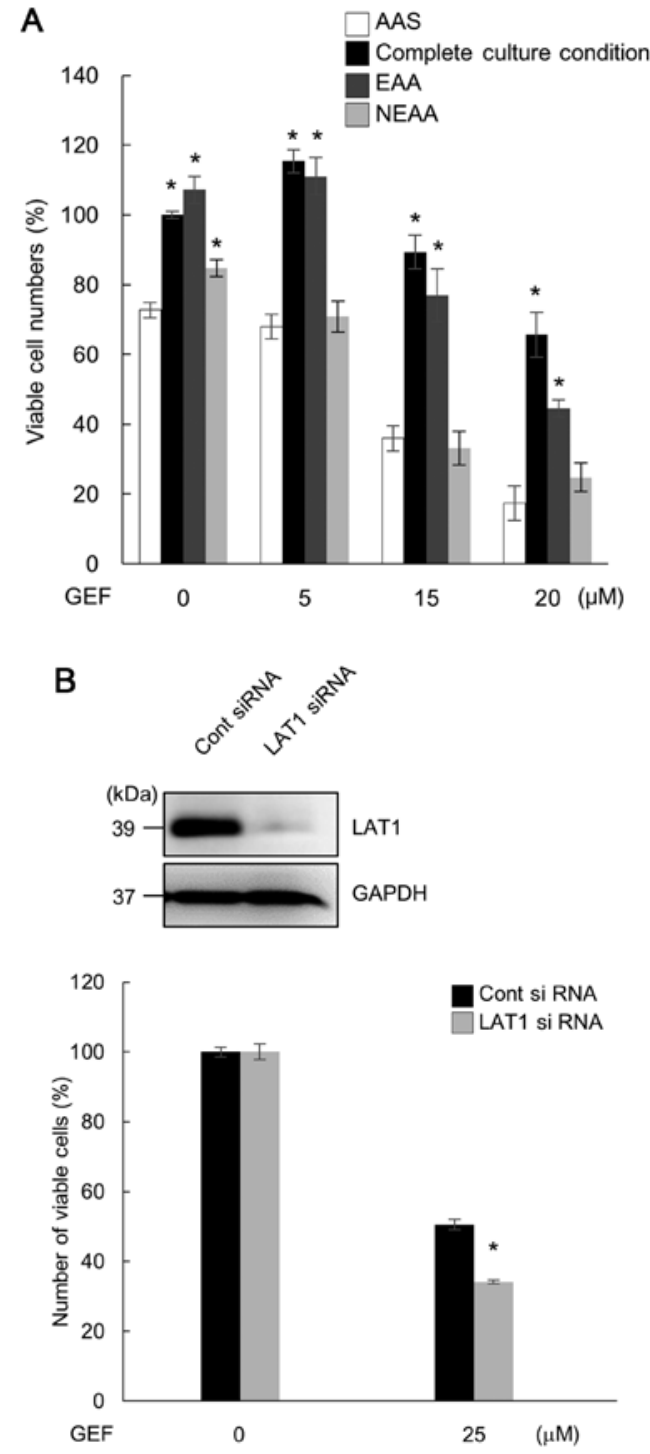

Figure 2. Shortage of intracellular amino acid pool is involved in the pronounced cytotoxicity of gefitinib (GEF) under amino acid starvation (AAS) culture conditions in CAL 27 cells. (A) CAL 27 cells were cultured under AAS conditions supplemented with/without essential and non-essential amino acids in the presence of GEF $(0-20 \mu \mathrm{M})$ for $48 \mathrm{~h}$. Viable cell number was assessed by CellTiter-Blue cell viability assay ("P<0.05, vs. AAS). EAA, essential amino acids; NEAA, non-essential amino acids. (B) Following pretreatment with LAT-1 siRNA or control siRNA for $24 \mathrm{~h}$, the CAL 27 cells were further cultured with/without GEF $(25 \mu \mathrm{M})$ for $48 \mathrm{~h}$ in DMEM containing $10 \%$ FBS, and viable cell numbers were assessed. Immunoblotting with anti-LAT-1 anibody (Ab) was performed after $24 \mathrm{~h}$ of treatment with siRNAs. Immunoblotting with anti-GAPDH monoclonal antibody $(\mathrm{mAb})$ was performed as an internal loading control; " $\mathrm{P}<0.05$, vs. control siRNA.

complete culture medium conditions or culture of the cells in AAS medium without GEF. Unlike staurosporine treatment for the typical induction of apoptosis, the number of Annexin $\mathrm{V}^{+} / \mathrm{PI}^{-}$cells indicating the cells undergoing an early phase of apoptosis was not increased (Fig. 3B). It was recently reported that phosphatidylserine labeled with Annexin $\mathrm{V}$ is exposed to the cell surface prior to loss of cell integrity during necroptosis induction (34). Therefore, Annexin V positive staining does not necessarily indicate cells undergoing apoptosis. As regards the morphological findings, we could not observe any chromatin condensation and nuclear fragmentation under AAS culture conditions plus GEF treatment, whereas cells treated with staurosporine exhibited the typical apoptotic karyorrhexis and blebbing of the plasma membrane. However, we observed the translucent cytoplasm and rupture of the plasma membrane of CAL 27 cells (Fig. 3C). These results indicate that non-apoptotic cell death appeared to be involved in the pronounced cytotoxicity induced by AAS culture conditions and GEF treatment.

Induction of autophagy in response to GEF and AAS conditions. We then examined whether autophagy is induced in response to GEF under either complete culture conditions or AAS culture conditions by immunoblotting with anti-LC3B $\mathrm{Ab}$ and anti-p62 Ab. As shown in Fig. 4A, the expression of p62, a substrate of autophagy, was decreased in response to $24 \mathrm{~h}$ of treatment with GEF in complete culture medium compared with that in the cells cultured without GEF in complete culture medium as a control (lane 1 vs. 2). p62 expression (lane 3) decreased in response to AAS without GEF and further decreased in response to $24 \mathrm{~h}$ of exposure to GEF plus AAS (lane 4). These results indicate that autophagy was accelerated by GEF under AAS culture conditions (Fig. 4A). In the CAL 27 cells, the expression ratio of LC3B-II to $\beta$-actin, a hallmark of autophagosome formation (35), was increased even under the complete culture condition (lane 1). This suggests the upregulation of autophagy under the normal culture condition in this cell line, which was consistent with the electron microscopic findings with an increased number of autophagosomes even in the control culture condition (data not shown). This was possibly due to the fact that the CAL 27 cells expressed a higher level of EGFR (Fig. 1A), implying a sufficient number of endosomal EGFR for autophagy induction (16). However, as regards the expression ratio of LC3B-II to $\beta$-actin, the CAL 27 cells under AAS culture conditions exhibited a decrease in this ratio compared with the control cells (lane 1 vs. 3). In addition, both the LC3B-I and LC3B-II bands became faint under AAS culture conditions, although AAS should induce more autophagy. We previously observed the same phenomenon when the cells had markedly upregulated autophagy along with leading LC3B-II degradation (18). As the expression level of LC3B-II is determined by the balance of synthesis and breakdown, the degradation of LC3B-II appeared to occur more rapidly than the induction of LC3B-II under AAS culture conditions. To confirm the accelerated induction of autophagy under AAS culture conditions plus GEF treatment, we carefully monitored autophagosome formation during a shorter exposure to GEF in either complete culture condition or AAS culture conditions using the A549 and CAL 27 cells, which stably express the tandem fluorescent-tagged LC3B (mCherry-EGFP-LC3B) by confocal microscopic time-lapse imaging (30). The number of fluorescent puncta indicating autophagosomes increased when the cells were cultured in the AAS medium in the absence of GEF. Both cell lines treated with GEF in the complete culture medium exhibited a greater number of mCherry-EGFP-puncta than the cells cultured in the AAS medium (Fig. 4B). The turnover of puncta appeared to be rapid, and most dots disappeared within 20 min after formation (data not shown). When the cells began to be cultured in the presence of GEF under AAS culture conditions, we observed a marked enhancement of autophagosome formation even within $30 \mathrm{~min}$ (Fig. 4B). Therefore, the 
A

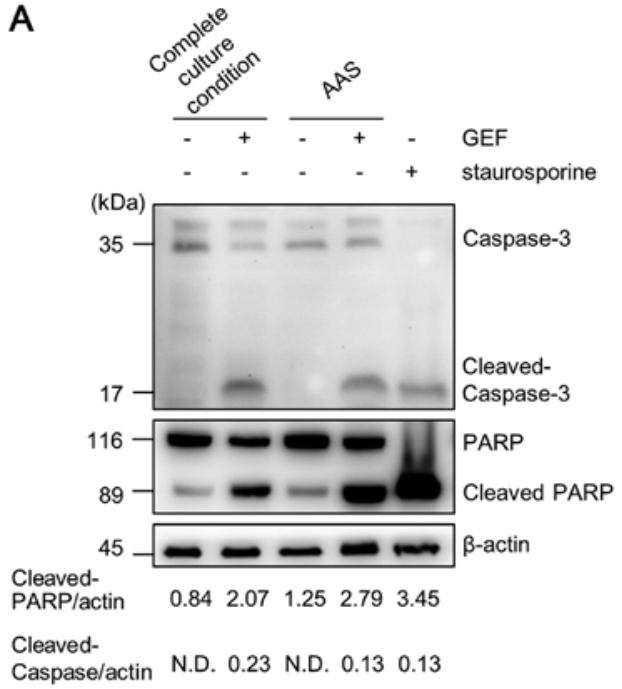

C
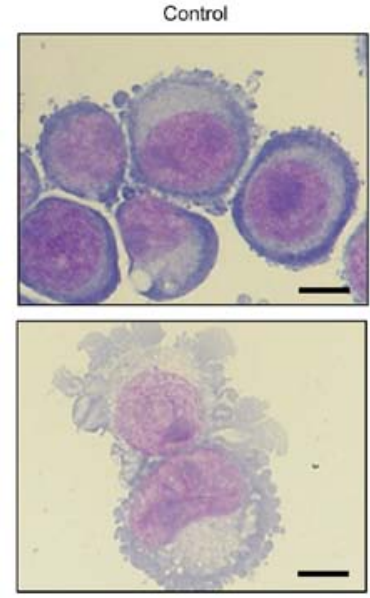

B

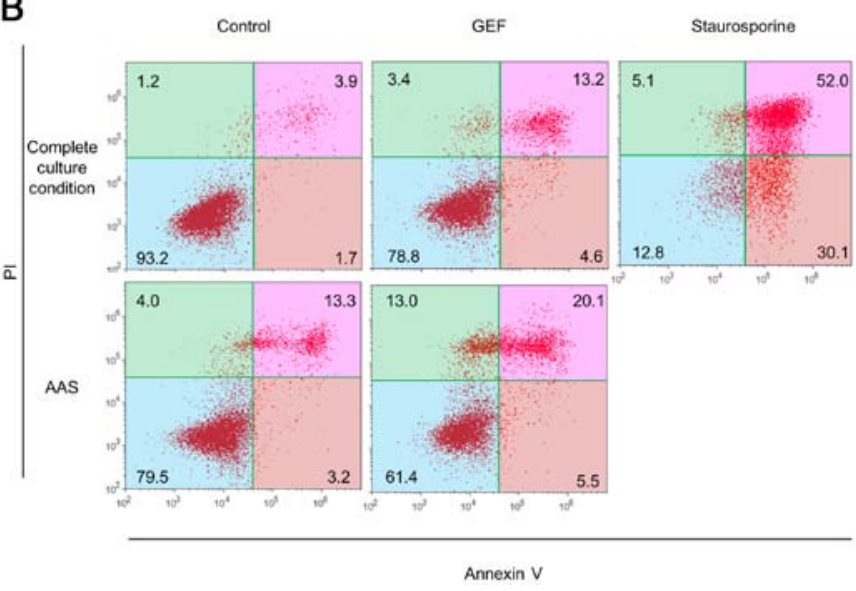

GEF
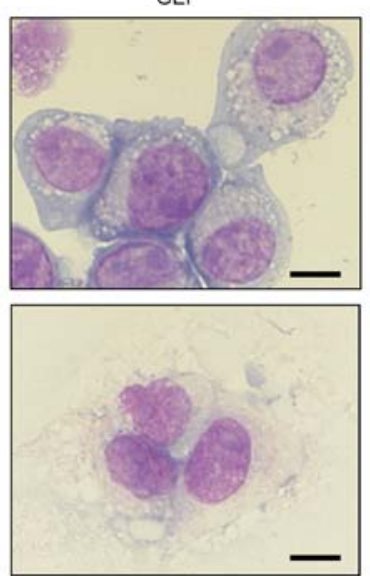

Figure 3. Non-apoptotic cell death induction following treatment with gefitinib (GEF) under amino acid starvation (AAS) conditions in CAL 27 cells. (A) Immunoblotting with caspase-3 antibody (Ab) and PARP Ab from the cell lysates of CAL 27 cells cultured under the complete culture condition or AAS with/without GEF $(25 \mu \mathrm{M})$ for $24 \mathrm{~h}$. Immunoblotting with anti- $\beta$-actin mAb was used as an internal control. Numbers indicate the ratios of the cleaved PARP to $\beta$-actin and the cleaved caspase-3 to $\beta$-actin in each lane. N.D., not detectable. (B) Flow cytometry with Annexin V/PI double staining after 24 h of treatment of CAL 27 cells with GEF $(25 \mu \mathrm{M})$ under the complete culture condition or AAS containing $10 \%$ FBS. The vertical axis indicates the log fluorescence intensity of propidium iodide (PI) and the horizontal axis indicates the log fluorescence intensity of Annexin V. The numbers indicate the percentage of cells in each area. (C) May-Grünwald-Giemsa staining was performed following treatment with/without GEF ( $25 \mu \mathrm{M})$ under the complete culture condition or AAS for $24 \mathrm{~h}$. As a positive control for apoptosis induction, CAL 27 cells were treated with staurosporine at $1 \mu \mathrm{M}$ for $4 \mathrm{~h}$. Scale bar, $10 \mu \mathrm{m}$.

induction of autophagy is accelerated in response to GEF plus AAS.

Induction of atypical necroptosis after treatment with GEF under AAS culture conditions. To examine whether the enhanced cytotoxicity is due to induction of 'autophagic cell death' (22,23), we used the Atg5 tet-off MEF cell line, named m5-7 (27). This cell line can be conditionally transformed into knockout the Atg5 gene, as a useful system for investigating the effects of autophagy in our study. Additionally, as MEF cells express EGFR, we intended to investigate whether our findings in cancer cell lines can be extended to immortalized fibroblasts.

Pre-treatment of the m5-7 cells with Dox, which leads to Atg5 knockout, results in the inhibition of autophagy (27). As shown in Fig. 5, the pronounced cytotoxicity by GEF $(50 \mu \mathrm{M})$ plus AAS was observed regardless of the deletion of the autophagic process in the m5-7 cells.
To investigate the molecular mechanisms responsible for this pronounced cell killing effect, we further examined the type of cell death involved in the GEF plus AAS treatment using CAL 27 cells. The cells were cultured with GEF either in the control medium or the AAS culture medium in the presence or absence of various inhibitors: 3-Methyl adenine (3-MA) for blocking autophagosome formation, SP600125 for blocking autophagic cell death (36), Z-VAD-fmk for inhibiting pan-caspases for blocking apoptosis, and NEC-1, a RIPK-1 inhibitor for blocking necroptosis (24). As shown in Fig. 6, treatment with 3-MA and SP600125 enhanced the cytotoxic effects of GEF and AAS possibly due to the cytotoxicity of the inhibitors. Taking the results of these inhibitors and m5-7 cells shown in Fig. 5 together, autophagic cell death appeared to be excluded. Z-VAD-fmk attenuated the GEF-induced cytotoxic effect, but did not exert any effects on the pronounced cytotoxicity of treatment with GEF plus AAS. Notably, in the presence of NEC-1, the pronounced cytotoxicity was almost completely 
A

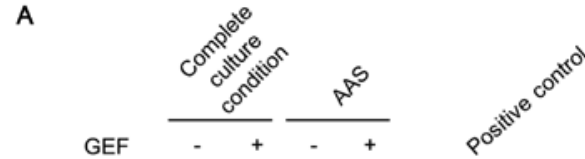
$(\mathrm{kDa})$

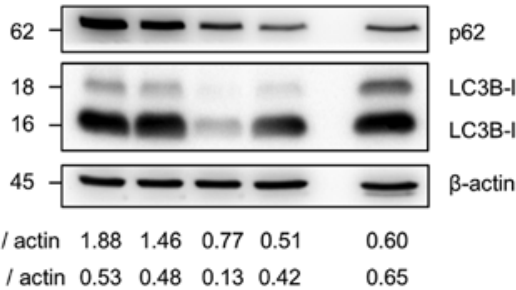

B
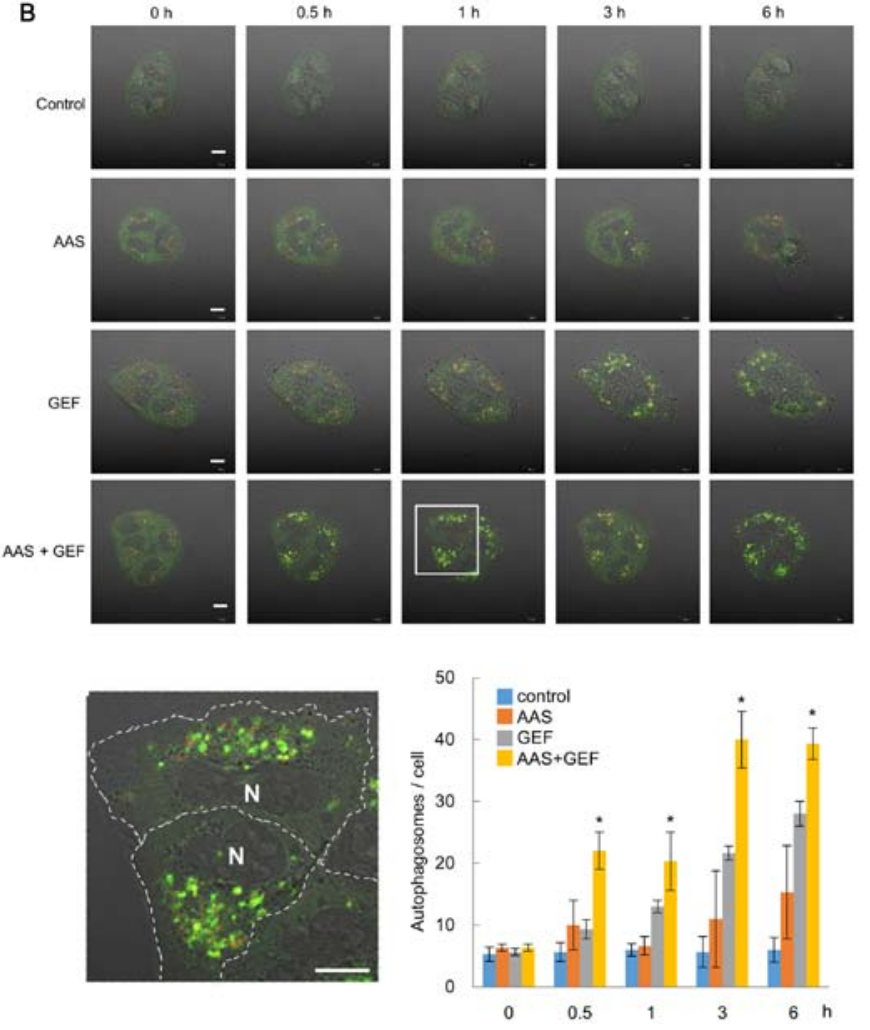

Figure 4. Induction of autophagy following treatment with gefitinib (GEF) under amino acid starvation (AAS) conditions or complete culture condition. (A) CAL 27 cells were treated with GEF at $25 \mu \mathrm{M}$ for $24 \mathrm{~h}$. Cellular proteins were separated by $15 \%$ SDS-PAGE, and immunoblotting was performed using anti-LC3B antibody (Ab) and anti-p62 monoclonal antibody $(\mathrm{mAb})$. Immunoblotting with anti- $\beta$-actin $\mathrm{mAb}$ was performed as an internal control. As a positive control for p62 and LC3B immunoblotting, the cell lysate derived from MDA-MB-231 cells treated with AZM $(50 \mu \mathrm{M})$ was usedas described in our literature (29). Numbers indicate the ratios of the p62 to $\beta$-actin and the LCB-II to $\beta$-actin in each lane. (B) Assessment of the induction of autophagy in A549/pBABE-puro-mCherry-EGFP-LC3B stable transfectants. Following $24 \mathrm{~h}$ of culture under the complete culture condition to make the cells adherent, the cells were washed twice with PBS and incubated in either the AAS culture medium containing 10\% FBS or control complete DMEM with/without GEF $(25 \mu \mathrm{M})$ for time-lapse imaging at 5-min interval by confocal laser scanning microscopy. Images were merged with EGFP, mCherry and phase contrast. Scale bar, $10 \mu \mathrm{m}$. The image on the left bottom panel is an enlarged image of the cells treated with AAS plus GEF at the 1-h time-point. N, nucleus. The numbers of intracellular autophagosome were assessed by counting the fluorescent puncta (EGFP and/or mCherry alone) in 20 cells in each culture condition, and plotted at the indicated timepoint. Data are representative of three independent experiments and values are expressed as the means \pm SEM ( ${ }^{\mathrm{P}}<0.05$, AAS plus GEF vs. GEF or AAS alone). CAL 27/pBABE-puro-mCherry-EGFP-LC3B stable transfectants behaved similarly to A549 transfectants in autophagosome formation. However, the precise assessment of autophagosome number was difficult on live imaging as the autophagosomes grow in a lump without forming a fine single cell layer (data not shown).
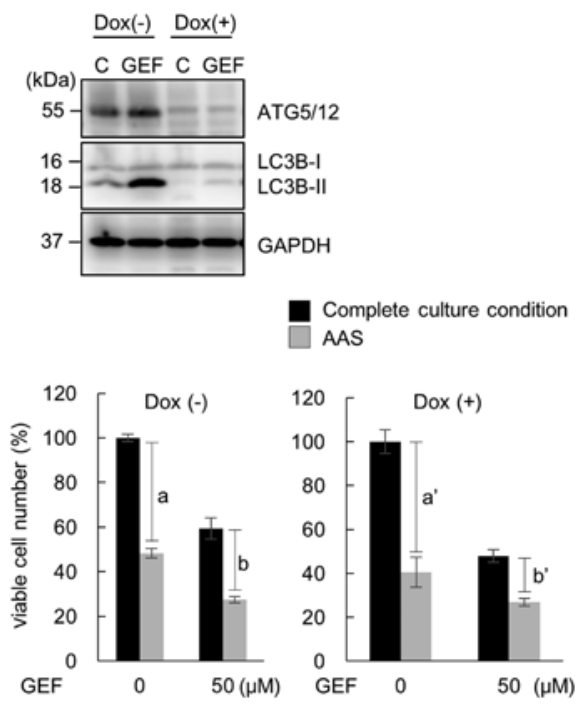

Figure 5. Effects of autophagy inhibition on gefitinib (GEF)-induced cytotoxicity using the Atg5 tet-off MEF cell line (m5-7). Following pre-treatment with/without doxycycline (Dox, $10 \mathrm{ng} / \mathrm{ml}$ ) for 4 days, the m5-7 cells were seeded in a 96-well culture plate in pentaplicate for $24 \mathrm{~h}$ and washed twice with PBS. The cell culture medium was replaced with complete culture medium or amino acid starvation (AAS) culture medium in the presence or absence of GEF ( $50 \mu \mathrm{M}$, at approximately $\left.\mathrm{IC}_{50}\right)$ for $24 \mathrm{~h}$. Assessment of the viable cell number and immunoblotting with anti-Atg5 antibody were performed as described above. Immunoblotting with GAPDH monoclonal antibody was used as an internal control. Significance is indicated as follows: a vs. a' shows no statistical significance, and b vs. b' also shows no statistical significance.

cancelled. This cytotoxicity cancellation was also observed in the A549 cells treated with GEF and AAS. Therefore, the induction of necroptosis in response to culture under AAS conditions plus GEF treatment was strongly suggested.

The CAL 27 cells adhere to the culture flask bottom in the normal culture condition, and they become non-adherent while undergoing cell death. As regards electron microscopy, the non-adherent CAL 27 cells treated with GEF plus AAS exhibited a translucent cytoplasm, cytoplasmic vacuoles, mitochondrial swelling, and plasma membrane rupture with an increased number of autophagosomes (Fig. 7). As similarly shown in Fig. 3C, no nuclear fragmentation, chromatin condensation, or apoptotic bodies were observed. Therefore, all these morphological findings also support the induction of necroptosis.

It is now well known that necroptosis is a RIPK-1/3dependent form of cell death (38-40). In TNF- $\alpha$-induced necroptosis, RIPK-1 is activated in response to TNF- $\alpha$ stimuli and forms a cytosolic complex, known as the necrosome, which is formed by RIPK-1 in complex with RIPK3 and MLKL $(39,40)$. MLKL is recruited to the necrosome and phosphorylated by RIPK3. This allows MLKL to form the MLKL oligomer for insertion into the plasma membrane to induce necroptosis $(41,42)$. Therefore, in this study, we knocked down RIPK-1 in CAL 27 cells using siRNA. The knockdown of RIPK-1 led to a significant attenuation of GEF plus AAS-induced cytotoxicity compared with the cells transfected with control siRNA (Fig. 8A). No differences were observed between the control siRNA- and RIPK-1 siRNA-treated cells under the GEF-containing culture conditions and AAS conditions. However, we could not detect the 

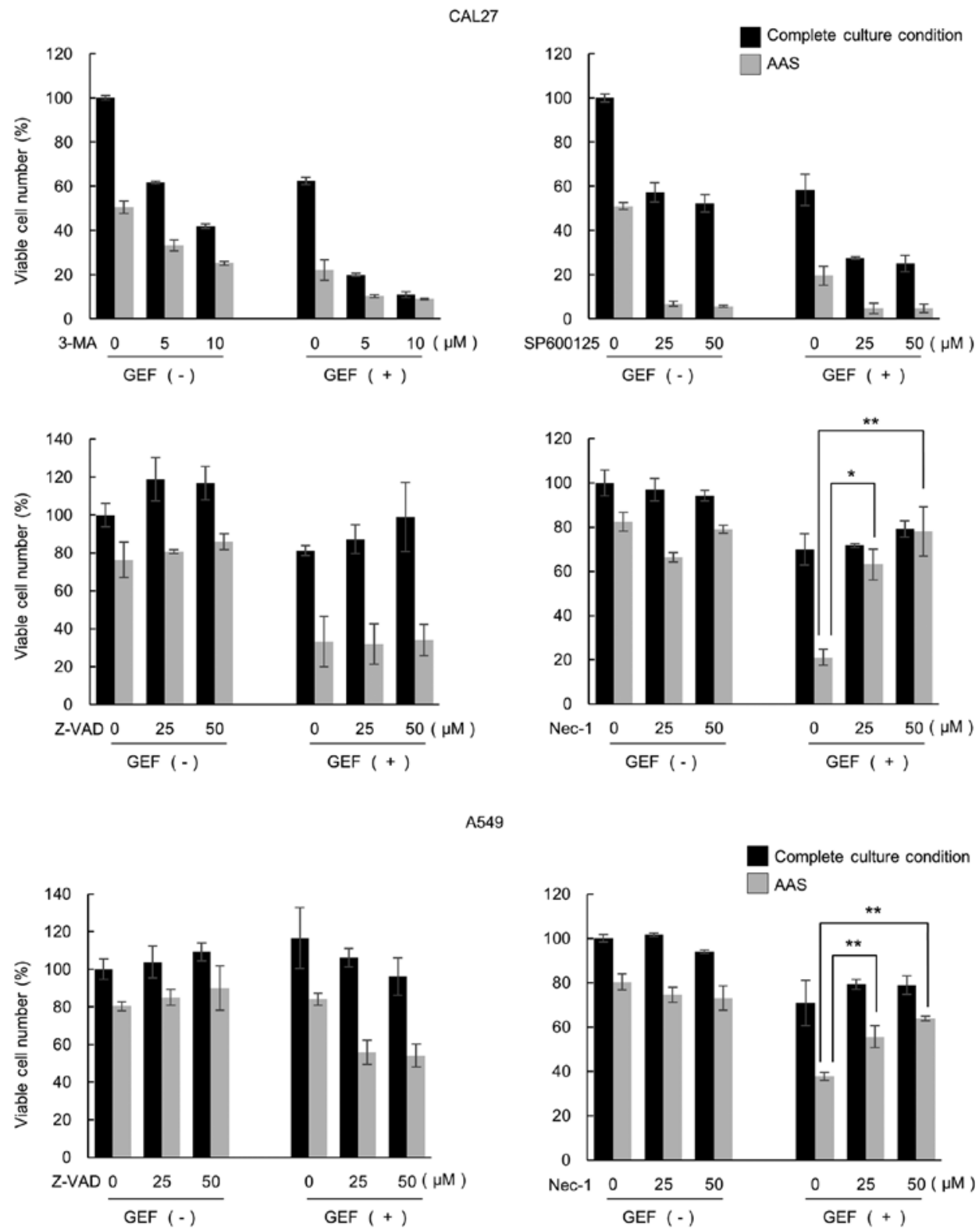

Figure 6. Effects of various inhibitors on the enhanced cytotoxicity by combining gefitinib (GEF) and amino acid starvation (AAS) in CAL 27 and A549 cells The CAL 27 and A549 cells were treated with 3-MA, SP600125, Z-VAD-fmk and necrostatin-1 (NEC-1) at the indicated concentrations in the presence or absence of GEF $(25 \mu \mathrm{M})$ under the complete culture condition or AAS for $24 \mathrm{~h}\left({ }^{*} \mathrm{P}<0.05\right.$ and ${ }^{* *} \mathrm{P}<0.01$, vs. AAS without NEC-1).

phosphorylation of RIPK-1 and MLKL by immunoblotting using specific Abs, which are known to be involved in the signaling pathways of TNF $\alpha$-induced necroptosis $(40,41)$ (Fig. 8B). In the immunoprecipitation assay using anti-RIPK-3 Ab, HT-29 cells pre-treated with Z-VAD-fmk followed by an additional treatment with cycloheximide and human TNF- $\alpha$ for the induction of necroptosis showed the co-precipitated phosphorylated (p)-MLKL, as well as p-RIPK-1; however, we could not detect the co-precipitated p-MLKL and p-RIPK-1 in the CAL 27 cells treated with GEF plus AAS (Fig. 8C). This suggested that culture under AAS conditions plus GEF treatment did not induce necrosome formation in the CAL 27 cells.

Taking all the data together, although the morphological findings and the effect of NEC-1 fit well with the induction of necroptosis, it would be more appropriate to describe the phenomenon as follows: The enhanced killing effect by the combined treatment with GEF plus AAS is mediated by 'atypical necroptosis' or alternatively, 'necroptosis-like cell death' in the CAL 27 cells.

\section{Discussion}

To the best of our knowledge, this is the first report showing that AAS sensitizes the killing effect of GEF in EGFRexpressing cancer cell lines (Fig. 1B). As a solid tumor appears to proliferate by adapting to nutrient insufficiency along with angiogenesis and autophagy, this has important clinical implications, such as the application of combination therapy of EGFR-TKI and LAT-1 inhibitors or anti-angiogenesis therapy for cancer patients. Indeed, it was previously reported that the phase II clinical trial of the anti-vascular endothelial growth factor monoclonal antibody bevacizumab in combination with erlotinib for advanced EGFR mutation-positive NSCLC resulted in an encouraging antitumor activity, and 


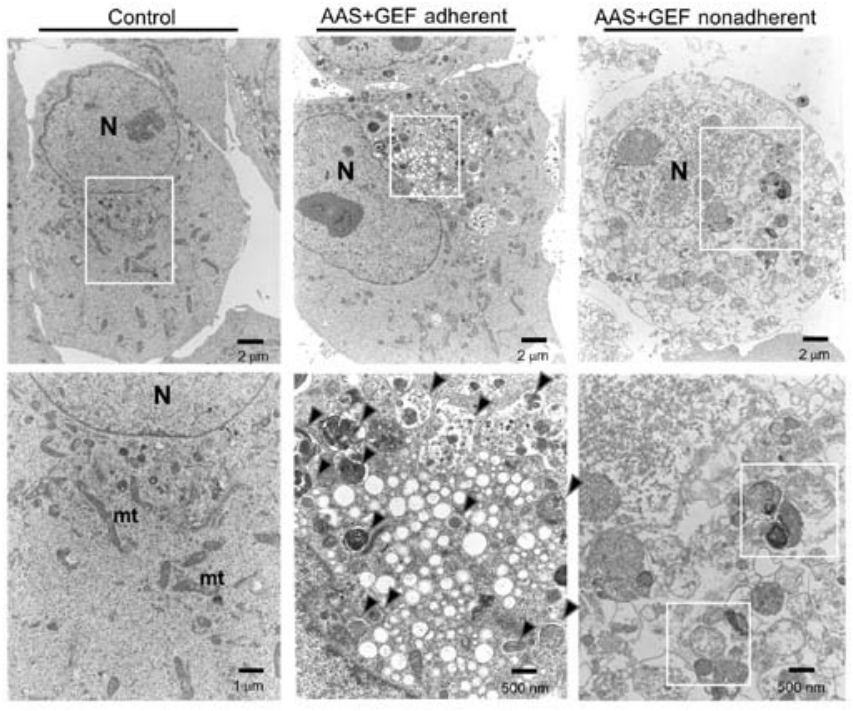

$\underline{\text { AAS+GEF nonadherent }}$

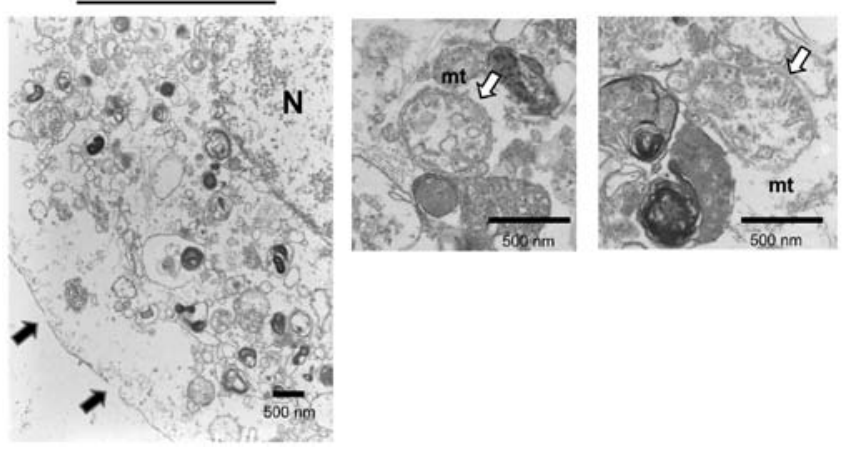

Figure 7. Electron microscopy of CAL 27 cells following treatment with gefitinib (GEF) under amino acid starvation (AAS) conditions. The CAL 27 cells were treated with GEF $(25 \mu \mathrm{M})$ under AAS culture conditions for $24 \mathrm{~h}$, and electron microscopy was performed in adherent and non-adherent CAL 27 cells. Scale bar represents the length indicated at each magnification. The white square box area in the upper panels was enlarged in the lower panels. $\mathrm{N}$, nucleus; mt, mitochondria; arrowhead, autophagosome/autolysosome; open (white) arrow, swollen mitochondria; closed (black) arrow, membrane rupture.

this could be a new first-line regimen for EGFR mutationpositive NSCLC $(43,44)$. In addition, we previously reported that macrolide antibiotics, which have an inhibitory effect on autophagy, exerted a CHOP/GADD153-mediated cell killing effect under amino acid starvation conditions in head and neck squamous cell carcinoma cell lines (18). As shown in Fig. 2A, the addition of amino acids to the culture medium resulted in the almost complete cancellation of cytotoxicity, implying that the cell killing effect appeared to be mediated by the shortage of intracellular amino acids owing to the blockage of their recycling process. Therefore, 'tumor-starving therapy' may be a novel concept for cancer therapy.

However, as shown in Fig. 1B, the response to AAS culture in terms of cell growth inhibition differed significantly in each cell line (e.g., the pancreatic cancer cell line, PANC-1, exhibited potent cytotoxicity in response to $48 \mathrm{~h}$ of AAS culture compared with other cancer cell lines). In addition, there was the significant difference in cell death under AAS conditions in the MEF cell line used in Fig. 1B and in m5-7, an Atg5 tet-off MEF cell line used in Fig. 5. The m5-7 cell line was generated by Hosokawa et al (27), and has been cloned for the complete inhibition of the autophagy machinery. During this cloning process, the m5-7 cell line appeared to have acquired a different phenotype including its response to AAS treatment compared with those in the immortalized MEF cell line. Therefore, the demand for intercellular amino acid pool appears to be varied among the cell lines, which is possibly due to the difference of cellular metabolism.

We deduced that the enhanced cell killing effect by GEF plus AAS was mediated by the induction of apoptosis. However, we could not detect any signs of enhanced apoptosis in the CAL 27 cells during the pronounced cytotoxicity (Figs. 3 and 7). Treatment with GEF alone induced caspase-3 and PARP cleavage to a certain extent, but much less than that induced by staurosporine (Fig. 3A). As the CAL 27 cells treated with staurosporine exhibited typical apoptotic features, such as PARP/caspase-3 cleavage, an increased number of the Annexin ${ }^{+} \mathrm{PI}^{-}$cell population as shown by flow cytometry, and morphological changes showing nuclear fragmentation and chromatin condensation (Fig. 3), the canonical machinery for apoptosis execution should be conserved in this cell line. The question that remains to be answered is which type of cell death phenotype was observed in this study and what cellular signals determine this phenotype.

According to the results shown in Figs. 3 and 6, autophagic cell death can be excluded in this case. The enhanced cytotoxicity shown in this study fits well with the necroptosis definition, that is, the morphological features of cell swelling and plasma membrane integrity, without chromatin condensation and nuclear fragmentation, and cell death inhibition by NEC-1 (Figs. 3, 6 and 7). The knockdown of RIPK-1 by siRNA, which significantly attenuated the enhanced cytotoxicity, also supports the induction of necroptosis (Fig. 8A). However, recent reports have revealed the molecular mechanism of necroptosis $(40,41)$ as follows: Various stresses that induce necroptosis appear to be mediated by the interaction of activated RIPK3 and MLKL. MLKL is a substrate for RIPK3 kinase activity, and MLKL phosphorylation at Thr357 and Ser358 by RIPK3 results in tetramer formation via the fourhelical bundle domain (4HBD) in the N-terminal region. This oligomerization subsequently leads to MLKL migration to the plasma membrane to bind phosphatidyl inositol lipids (42). MLKL tetramer accumulation in the membrane site finally forms an octamer and acts as the cation channel for ion influx, which increases the intracellular osmotic pressure leading to cellular destruction (45). As shown in Fig. 4B, autophagy was considerably upregulated in response to GEF and AAS. It has recently been reported that, in association with the autophagic machinery for TRAIL-induced necroptosis, p62/SQSTM1 recruits RIPK1 and mediates the formation of a multimolecular complex designated as necrosome consisting of RIPK1, RIPK3 and MLKL for the upregulation of RIPK3 kinase activity (46). Therefore, the rapid upregulation of autophagosomes in response to GEF plus AAS appears to function as a scaffold of necrosome formation, switching toward necroptosis rather than the induction of apoptosis. However, there were no signs of the phosphorylation of RIPK1 and MLKL, or necrosome formation (Fig. 8B and C). Although other unclarified machineries for necroptosis may still exist, these data indicate a non-standard execution of necroptosis. As the enhanced cytotoxicity by GEF plus AAS was significantly attenuated in 
A
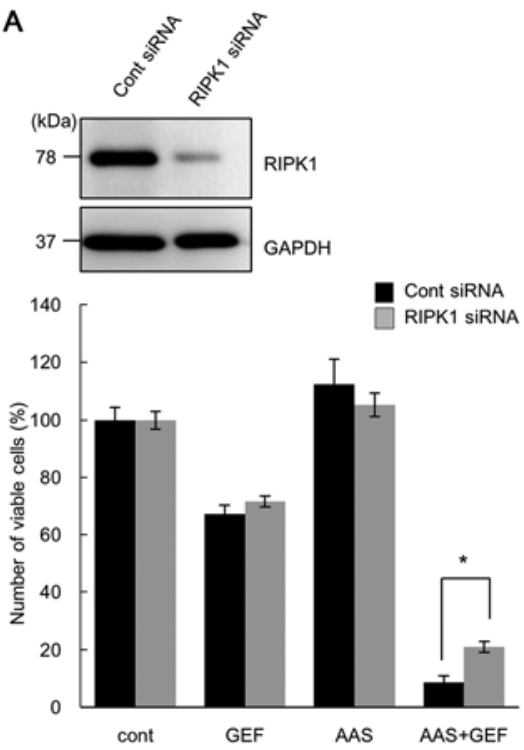

B

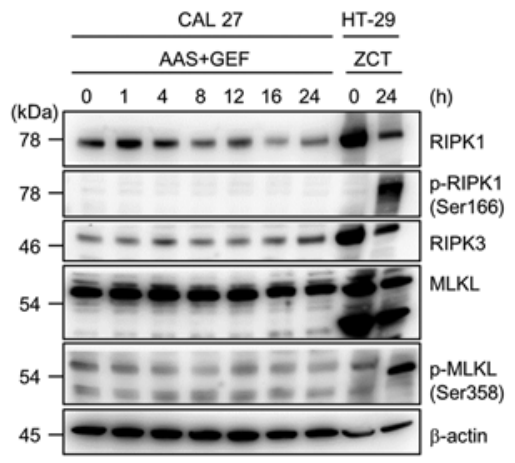

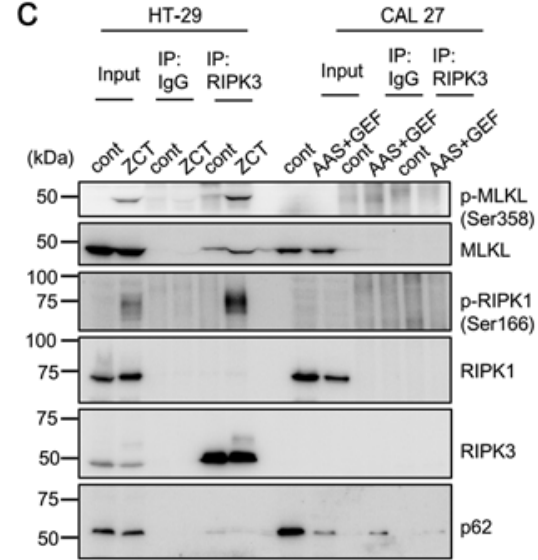

Figure 8. Assessment of the induction of necroptosis in CAL 27 cells following treatment with gefitinib (GEF) under amino acid starvation (AAS) conditions. (A) Following pre-treatment with RIPK1 siRNA or control siRNA for $48 \mathrm{~h}$, the CAL 27 cells were further cultured with/without GEF (25 $\mu$ M) under either AAS conditions with 10\% FBS or DMEM containing 10\% FBS, and viable cell number was assessed. Immunoblotting with anti-RIPK1 antibody was performed after $24 \mathrm{~h}$ of treatment with siRNAs. Immunoblotting with anti-GAPDH monoclonal antibody was performed as an internal loading control ( ${ }^{*} \mathrm{P}<0.05$, control siRNA vs. RIPK1 siRNA). (B) Following treatment of the CAL 27 cells with GEF ( $\left.25 \mu \mathrm{M}\right)$ under AAS culture conditions for the indicated time periods, the cells were lysed and the cellular proteins were separated by SDS-PAGE, and then immunoblotted with anti-RIPK1 antibody (Ab), anti-RIPK3 $\mathrm{Ab}$, anti-MLKL Ab, and phosphor (p)-specific Abs for RIPK1 MLKL Abs. As for the positive control for the typical necroptosis induction, HT-29 cells were pre-treated with Z-VAD-fmk $(20 \mu \mathrm{M})$ for $30 \mathrm{~min}$ followed by an additional treatment with cycloheximide $(\mathrm{CHX}, 10 \mu \mathrm{g} / \mathrm{ml})$ and human TNF- $\alpha(20 \mathrm{ng} / \mathrm{ml})$ for $8 \mathrm{~h}$ as previously reported (28). This treatment is indicated as ZCT. (C) The CAL 27 cells treated with GEF (25 $\mu \mathrm{M})$ under AAS conditions for $24 \mathrm{~h}$ were used for co-immunoprecipitation assay. The CAL 27 cells treated under the complete culture condition without GEF were used as control. Cell lysates were immunoprecipitated with anti-RIPK3 Ab or the isotype matched IgG. Immunoprecipitates were separated by SDS-PAGE and immunoblotted with either anti-p-MLKL, anti-MLKL, anti-p-RIPK1, anti-RIPK1, anti-RIPK3, or anti-p62 Ab. HT-29 cells treated with ZCT as described above were used as positive control for necrosome formation.

the presence of NEC-1, as well as the knockdown of RIPK1, this cell death phenotype appears to be dependent on RIPK1, at least in part (Figs. 6 and 8A). The RIPK3 expression level was much lower in the CAL 27 cells than in the HT-29 cells (Fig. 8B). Thus, the lower RIPK3 expression level in CAL 27 cells may affect the execution of necroptosis in our case.

In contrast to necroptosis acceleration, autophagy has been reported to rather suppress necroptosis in RCC4 cells, a human renal cell carcinoma cell line (47). The inhibition of mTOR with CCL-779, a specific mTOR inhibitor, has been shown to stimulate autophagy and lead to the elimination of RIPKs via autophagy-mediated degradation (47). The simultaneous inhibition of mTOR with CCL-779 and autophagy with chloroquine resulted in necroptosis induction. Autophagy promotes the apoptosis induced by Fas ligand/CD95 due to its ability to degrade Fap-1, a negative regulator of CD95 signaling (48). On the other hand, autophagy protects the apoptosis induced by TNF- $\alpha$ by controlling the levels of PUMA, a pro-apoptotic BCL-2 family protein (49). As shown in Fig. 8B, RIPK1 expression was downregulated during the 24-h exposure to AAS plus GEF in the CAL 27 cells. Therefore, during the upregulated autophagic degradation, the delicate balance of the molecular components for cell death execution may also determine the cell fate and cell death phenotype.

It has recently been reported that when the MLKL activation is limited or reversed, the ESCRT-III machinery controls the duration of plasma membrane integrity, which may exert an efficient in vivo immune response (34). In contrast to apoptosis, necrosis and necroptosis induce a series of immunological responses resulting in inflammation in vivo. Therefore, a more fine-tuned system consisting of many regulatory molecules might exist. Modulation of the necroptotic process by degradation of regulators and effectors may have some unknown biological roles including tumor immunity. Although the precise molecular mechanism for executing the pronounced cell killing effect in our case remains to be clarified, the varying amounts of degradation of molecular components for cell death execution might interfere with the cell death phonotype, but 'not in a stereotypical manner'.

\section{Acknowledgements}

We would like to thank Professor Noboru Mizushima (University of Tokyo, Tokyo, Japan) for kindly providing us with the tet-off Atg5 m5-7 cell line. We would also like to thank Dr Edward Barroga (http://orcid.org/0000-00028920-2607), Associate Professor and Senior Medical Editor of Tokyo Medical University for editing the manuscript. This study was supported by funds provided through a MEXTsupported program of the Strategic Research Foundation at Private Universities (S1411011, 2014-2018) from the Ministry of Education, Culture, Sports, Science and Technology of Japan, and partially supported by JSPS KAKENHI Grant Numbers JP26460478, JP16K19187, JP16K21388, and JP16K21387, and a Grant-in-Aid from Tokyo Medical University Cancer Research to N.T. 


\section{Competing interests}

The authors declare that they have no competing interests.

\section{References}

1. Kuma A and Mizushima N: Physiological role of autophagy as an intracellular recycling system: With an emphasis on nutrient metabolism. Semin Cell Dev Biol 21: 683-690, 2010.

2. Segawa H, Fukasawa Y, Miyamoto K, Takeda E, Endou H and Kanai Y: Identification and functional characterization of a $\mathrm{Na}^{+}$-independent neutral amino acid transporter with broad substrate selectivity. J Biol Chem 274: 19745-19751, 1999.

3. Yanagida O, Kanai Y, Chairoungdua A, Kim DK, Segawa H, Nii T, Cha SH, Matsuo H, Fukushima J, Fukasawa Y, et al: Human L-type amino acid transporter 1 (LAT1): Characterization of function and expression in tumor cell lines. Biochim Biophys Acta 1514: 291-302, 2001.

4. Oda K, Hosoda N, Endo H, Saito K, Tsujihara K, Yamamura M, Sakata T, Anzai N, Wempe MF, Kanai Y, et al: L-type amino acid transporter 1 inhibitors inhibit tumor cell growth. Cancer Sci 101: 173-179, 2010.

5. Sakata T, Ferdous G, Tsuruta T, Satoh T, Baba S, Muto T, Ueno A, Kanai Y,Endou H and Okayasu I: L-type amino-acid transporter 1 as a novel biomarker for high-grade malignancy in prostate cancer. Pathol Int 59: 7-18, 2009.

6. Mizushima N, Levine B, Cuervo AM and Klionsky DJ: Autophagy fights disease through cellular self-digestion. Nature 451: 1069-1075, 2008

7. Galluzzi L, Pietrocola F, Levine B and Kroemer G: Metabolic control of autophagy. Cell 159: 1263-1276, 2014.

8. White E: Deconvoluting the context-dependent role for autophagy in cancer. Nat Rev Cancer 12: 401-410, 2012.

9. Han W, Pan H, Chen Y, Sun J, Wang Y, Li J, Ge W, Feng L, Lin $\mathrm{X}$, Wang $\mathrm{X}$, et al: EGFR tyrosine kinase inhibitors activate autophagy as a cytoprotective response in human lung cancer cells. PLoS One 6: e18691, 2011.

10. Fung $\mathrm{C}$, Chen X, Grandis JR and Duvvuri U: EGFR tyrosine kinase inhibition induces autophagy in cancer cells. Cancer Biol Ther 13: 1417-1424, 2012

11. Sugita S, Ito K, Yamashiro Y, Moriya S, Che XF, Yokoyama T, Hiramoto $M$ and Miyazawa K: EGFR-independent autophagy induction with gefitinib and enhancement of its cytotoxic effect by targeting autophagy with clarithromycin in non-small cell lung cancer cells. Biochem Biophys Res Commun 461: 28-34, 2015.

12. Mukai S, Moriya S, Hiramoto M, Kazama H, Kokuba H, Che XF, Yokoyama T, Sakamoto S, Sugawara A, Sunazuka T, et al: Macrolides sensitize EGFR-TKI-induced non-apoptotic cell death via blocking autophagy flux in pancreatic cancer cell lines. Int J Oncol 48: 45-54, 2016.

13. Wei Y, Zou Z, Becker N, Anderson M, Sumpter R, Xiao G Kinch L, Koduru P, Christudass CS, Veltri RW, et al: EGFRmediated Beclin 1 phosphorylation in autophagy suppression, tumor progression, and tumor chemoresistance. Cell 154: $1269-1284,2013$

14. Sordella R, Bell DW, Haber DA and Settleman J: Gefitinibsensitizing EGFR mutations in lung cancer activate anti-apoptotic pathways. Science 305: 1163-1167, 2004.

15. Ciardiello F and Tortora G: EGFR antagonists in cancer treatment. N Engl J Med 358: 1160-1174, 2008

16. Tan X, Thapa N, Sun Y and Anderson RA: A kinase-independent role for EGF receptor in autophagy initiation. Cell 160: 145-160, 2015.

17. Moriya S, Che XF, Komatsu S, Abe A, Kawaguchi T, Gotoh A, Inazu M, Tomoda A and Miyazawa K: Macrolide antibiotics block autophagy flux and sensitize to bortezomib via endoplasmic reticulum stress-mediated CHOP induction in myeloma cells. Int J Oncol 42: 1541-1550, 2013

18. Hirasawa K, Moriya S, Miyahara K, Kazama H, Hirota A, Takemura J, Abe A, Inazu M, Hiramoto M, Tsukahara K, et al: Macrolide antibiotics exhibit cytotoxic effect under amino aciddepleted culture condition by blocking autophagy flux in head and neck squamous cell carcinoma cell lines. PLoS One 11: e0164529, 2016.

19. Kim YC and Guan KL: mTOR: A pharmacologic target for autophagy regulation. J Clin Invest 125: 25-32, 2015.
20. Kim J, Kundu M, Viollet B and Guan KL: AMPK and mTOR regulate autophagy through direct phosphorylation of Ulk1. Nat Cell Biol 13: 132-141, 2011.

21. Dalle Pezze P, Ruf S, Sonntag AG, Langelaar-Makkinje M, Hall P, Heberle AM, Razquin Navas P, van Eunen K, Tölle RC, Schwarz JJ, et al: A systems study reveals concurrent activation of AMPK and mTOR by amino acids. Nat Commun 7: 13254, 2016.

22. Kondo Y, Kanzawa T, Sawaya R and Kondo S: The role of autophagy in cancer development and response to therapy. Nat Rev Cancer 5: 726-734, 2005.

23. Shimizu S, Kanaseki T, Mizushima N, Mizuta T, ArakawaKobayashi S, Thompson CB and Tsujimoto Y: Role of Bcl-2 family proteins in a non-apoptotic programmed cell death dependent on autophagy genes. Nat Cell Biol 6: 1221-1228, 2004.

24. Teng X, Degterev A, Jagtap P, Xing X, Choi S, Denu R, Yuan J and Cuny GD: Structure-activity relationship study of novel necroptosis inhibitors. Bioorg Med Chem Lett 15: 5039-5044, 2005.

25. Degterev A, Huang Z, Boyce M, Li Y, Jagtap P, Mizushima N, Cuny GD, Mitchison TJ, Moskowitz MA and Yuan J: Chemical inhibitor of nonapoptotic cell death with therapeutic potential for ischemic brain injury. Nat Chem Biol 1: 112-119, 2005.

26. Kawahara A, Yamamoto C, Nakashima K, Azuma K, Hattori S, Kashihara M, Aizawa H, Basaki Y, Kuwano M, Kage M, et al: Molecular diagnosis of activating EGFR mutations in non-small cell lung cancer using mutation-specific antibodies for immunohistochemical analysis. Clin Cancer Res 16: 3163-3170, 2010.

27. Hosokawa N, Hara Y and Mizushima N: Generation of cell lines with tetracycline-regulated autophagy and a role for autophagy in controlling cell size. FEBS Lett 581: 2623-2629, 2007.

28. Sun XM, MacFarlane M, Zhuang J, Wolf BB, Green DR and Cohen GM: Distinct caspase cascades are initiated in receptormediated and chemical-induced apoptosis. J Biol Chem 274: 5053-5060, 1999.

29. Miyahara K, Kazama H, Kokuba H, Komatsu S, Hirota A, Takemura J, Hirasawa K, Moriya S, Abe A, Hiramoto M, et al: Targeting bortezomib-induced aggresome formation using vinorelbine enhances the cytotoxic effect along with ER stress loading in breast cancer cell lines. Int J Oncol 49: 1848-1858, 2016.

30. Hansen TE and Johansen T: Following autophagy step by step. BMC Biol 9: 39, 2011.

31. Golden SH, Folsom AR, Coresh J, Sharrett AR, Szklo M and Brancati F: Risk factor groupings related to insulin resistance and their synergistic effects on subclinical atherosclerosis: The atherosclerosis risk in communities study. Diabetes 51: 3069-3076, 2002

32. Lynch TJ, Bell DW, Sordella R, Gurubhagavatula S, Okimoto RA, Brannigan BW, Harris PL, Haserlat SM, Supko JG, Haluska FG, et al: Activating mutations in the epidermal growth factor receptor underlying responsiveness of non-small-cell lung cancer to gefitinib. N Engl J Med 350: 2129-2139, 2004.

33. Wang J, Chen X, Su L, Li P, Liu B and Zhu Z: LAT-1 functions as a promotor in gastric cancer associated with clinicopathologic features. Biomed Pharmacother 67: 693-699, 2013

34. Gong YN, Guy C, Olauson H, Becker JU, Yang M, Fitzgerald P, Linkermann A and Green DR: ESCRT-III acts downstream of MLKL to regulate necroptotic cell death and its consequences. Cell 169: 286-300.e16, 2017.

35. Klionsky DJ, Abdelmohsen K, Abe A, Abedin MJ, Abeliovich H, Acevedo Arozena A, Adachi H, Adams CM, Adams PD, Adeli K, et al: Guidelines for the use and interpretation of assays for monitoring autophagy (3rd edition). Autophagy 12: 1-222, 2016.

36. Chen L, Meng Y, Guo X, Sheng X, Tai G, Zhang F, Cheng H and Zhou Y: Gefitinib enhances human colon cancer cells to TRAILinduced apoptosis of via autophagy- and JNK-mediated death receptors upregulation. Apoptosis 21: 1291-1301, 2016.

37. Degterev A, Hitomi J, Germscheid M, Ch'en IL, Korkina O, Teng X, Abbott D, Cuny GD, Yuan C, Wagner G, et al: Identification of RIP1 kinase as a specific cellular target of necrostatins. Nat Chem Biol 4: 313-321, 2008

38. Weinlich R, Oberst A, Beere HM and Green DR: Necroptosis in development, inflammation and disease. Nat Rev Mol Cell Biol 18: 127-136, 2017.

39. Li J, McQuade T, Siemer AB, Napetschnig J, Moriwaki K, Hsiao YS, Damko E, Moquin D, Walz T, McDermott A, et al: The RIP1/RIP3 necrosome forms a functional amyloid signaling complex required for programmed necrosis. Cell 150: 339-350, 2012. 
40. Zhang Y, Su SS, Zhao S, Yang Z, Zhong CQ, Chen X, Cai Q, Yang ZH, Huang D, Wu R, et al: RIP1 autophosphorylation is promoted by mitochondrial ROS and is essential for RIP3 recruitment into necrosome. Nat Commun 8: 14329, 2017.

41. Ros U, Peña-Blanco A, Hänggi K, Kunzendorf U, Krautwald S, Wong WW and García-Sáez AJ: Necroptosis execution is mediated by plasma membrane nanopores independent of calcium. Cell Reports 19: 175-187, 2017.

42. Sun L, Wang H, Wang Z, He S, Chen S, Liao D, Wang L, Yan J, Liu W, Lei X, et al: Mixed lineage kinase domain-like protein mediates necrosis signaling downstream of RIP3 kinase. Cell 148: 213-227, 2012.

43. Seto T, Kato T, Nishio M, Goto K, Atagi S, Hosomi Y Yamamoto N, Hida T, Maemondo M, Nakagawa K, et al: Erlotinib alone or with bevacizumab as first-line therapy in patients with advanced non-squamous non-small-cell lung cancer harbouring EGFR mutations (JO25567): An open-label, randomised, multicentre, phase 2 study. Lancet Oncol 15: 1236-1244, 2014.

44. Ichihara E, Hotta K, Nogami N, Kuyama S, Kishino D, Fujii M, Kozuki T, Tabata M, Harada D, Chikamori K, et al: Phase II trial of gefitinib in combination with bevacizumab as first-line therapy for advanced non-small cell lung cancer with activating EGFR gene mutations: The Okayama Lung Cancer Study Group Trial 1001. J Thorac Oncol 10: 486-491, 2015.

45. Huang D, Zheng X, Wang ZA, Chen X, He WT, Zhang Y, Xu JG, Zhao H, Shi W, Wang X, et al: The MLKL channel in necroptosis is an octamer formed by tetramers in a dyadic process. Mol Cell Biol 37: e00497-e16, 2017.
46. Goodall ML, Fitzwalter BE, Zahedi S, Wu M, Rodriguez D, Mulcahy-Levy JM, Green DR, Morgan M, Cramer SD and Thorburn A: The autophagy machinery controls cell death switching between apoptosis and necroptosis. Dev Cell 37: 337-349, 2016.

47. Bray K, Mathew R, Lau A, Kamphorst JJ, Fan J, Chen J, Chen HY, Ghavami A, Stein M, DiPaola RS, et al: Autophagy suppresses RIP kinase-dependent necrosis enabling survival to mTOR inhibition. PLoS One 7: e41831, 2012.

48. Gump JM, Staskiewicz L, Morgan MJ, Bamberg A, Riches DW and Thorburn A: Autophagy variation within a cell population determines cell fate through selective degradation of Fap-1. Nat Cell Biol 16: 47-54, 2014.

49. Thorburn J, Andrysik Z, Staskiewicz L, Gump J, Maycotte P, Oberst A, Green DR, Espinosa JM and Thorburn A: Autophagy controls the kinetics and extent of mitochondrial apoptosis by regulating PUMA levels. Cell Rep 7: 45-52, 2014.

This work is licensed under a Creative Commons Attribution-NonCommercial-NoDerivatives 4.0 International (CC BY-NC-ND 4.0) License. 